\title{
EDITOR'S PAGE \\ Did spinal cord management change in the last 40 years
}

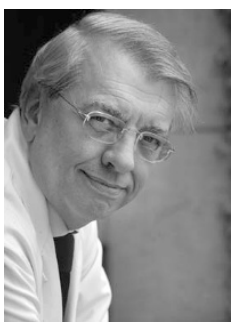

\author{
JJ Wyndaele \\ Antwerp, Belgium \\ E-mail: wyndaele@uza.be
}

I would like to start by quoting some literature.

"The idea of giving spinal cord sufferers a comprehensive treatment and care from the start and throughout all stages is now more and more universally recognized, as against the customary approach of fragmentation of the initial and early treatment of these patients to their social rehabilitation, erronously called "Third Phase" of management. In this respect, the congregation of paraplegics and tetraplegics in Spinal Centres under the care of a specialized staff has proved beyond all doubt to be the best basis for such comprehensive management of these most severely disabled victims of accidents and disease, and consequently the idea of setting up Spinal Injuries Centres, ...., is now spreading all over the world... One can point to progress in many directions, but some of the more outstanding may be mentioned here: the participation of the isolated cord through co-ordinated reflexes in the restoration and maintenance of the upright position of the paralysed, reorientation of postural and vasomotor control by special methods, prevention and treatment of pressure sores and infection of the urinary tract in the immediate and later stages, restoration of the respiratory function in tetraplegics and a new approach to the sexual problems. Our findings of the highly significant role played by autonomic mechanisms, especially the cardio-vascular system, in the function of the organisms below and above the transection of the spinal cord have opened new vistas into future research. Ways have been revealed in which the dysfunction of internal organs in the paralysed part of the body excite abnormal discharge of the various components of the autonomic system and also how the autonomic hyperreflexia can be controlled and avoided." Sir Ludwig Guttmann 1973.

Or some years later: "The epidemic of spinal paralysis is increasing internationally. Although the percentage of spinal paralysis in the total population remains small, this condition accounts for considerable human suffering and an unusually expenditure of hospital time and resources. In the past decade, practical methods of management have considerably reduced both the duration of hospitalization and related costs. A chapter on the paraplegic in developing countries demonstrates that even with limited financial resources, improvement of care can be achieved... particularly nursing and physical therapy, inevidently assumes the largest role in the book. All members of the medical team need to recognize that their involvement in this area must be steady and constant, requiring a major proportion of their time and effort. Restoration is not new: it is as old as medicine and has been the aim and object of medicine from its earliest days. There is noting magical about the care and management of paraplegics and tetraplegics-just hard work, which is time consuming and sometimes frustrating, but nevertheless successfull and rewarding." George Bedbrook 1981.

From the same year but from a different part of the world: "The methods used are based mainly on common sense, attention to details, perseverance and a stimulating goodwill. It is not surpring that paraplegia has been so much impregnated by the spirit of readaptation medicine, to the point of constituting one of its best examples. It has become almost a speciality in its own right, with its International Society, its journal, its medical associations in different countries and... paraplegia specialists in centres reserved for his type of disease. Certainly, it is not necessary that the person who directs such a centre should be a paraplegia specialised person, but the centre with the best equipment in the world would never become a real spinal cord injury centre if the one who runs it not animates and not infuses his collaborators the spirit of reeducation, with the humanity and the understanding that seem indispensable for the knowledge and the treatment related to each paraplegic." Free translation from French. Marc Maury 1981.

My conclusion is that the general requirement of SCI management has not changed in 40 years across the world. Moreover much has been achieved in further knowledge and that this process continues without hesitation. This issue comprises some excellent papers that emphasizes the above. These papers help further understand spasticity treatment, datasets, neuropathic pain, urological complications, financial burden, sleep disordered breathing AND MORE.

Spinal Cord (2015) 53, 83; doi:10.1038/sc.2015.1 